\title{
An Application of Statistics to Meteorology: Estimation of Motion
}

\section{David R. Brillinger ${ }^{1}$}

\begin{abstract}
Concern is with moving meteorological phenomena. Some existing techniques for the estimation of motion parameters are reviewed. Fourier-based and generalized-additive-model-based analyses are then carried out for the global geopotential 500 millibar (mb) height field during the period 1-6 January 1986.
\end{abstract}

\subsection{Introduction}

Early in his professional career Lucien Le Cam was a statistician at Electricité de France. His Fourth Berkeley Symposium paper, "A stochastic description of precipitation", (Le Cam 1961), describes a conceptual stochastic model for (perhaps moving) rainfields developed at that time. In particular, Professor Le Cam was concerned with the development of river stream flow following rainfall. The model involved a smoothing transformation of a point process with direction and velocity of movement included. There have since been many references building on his work including: (Smith \& Karr 1985), (Gupta \& Waymire 1987), (Cox \& Isham 1988), (Phelan 1992).

In this paper the focus is on velocity estimation of moving disturbances. To begin consider a number, $N$, of plane waves, $g_{n}\left(\alpha_{n} x+\beta_{n} y-v_{n} t\right)$, moving across a surface. The model of interest is

$$
Y(x, y, t)=\sum_{n=1}^{N} g_{n}\left(\alpha_{n} x+\beta_{n} y-v_{n} t\right)+\text { noise }
$$

with $(x, y)$ location, $t$ time and the $n-t h$ wave having velocity $v_{n}$ and direction cosines $\left(\alpha_{n}, \beta_{n}\right)$. The velocity is the parameter of particular interest in this work. The functions $g_{n}($.$) may be known up to a finite dimensional pa-$ rameter, eg. $g(u)=\rho \cos (m u+\phi)$ or simply may be assumed smooth. The

\footnotetext{
${ }^{1}$ University of California, Berkeley, USA
} 
first case suggests employing Fourier techniques, while the second suggests projection pursuit regression techniques to study the velocities.

The analyses presented here are for a worldwide spatial-temporal field. An interesting aspect is that, because the data are for the whole sphere, there is a basic periodicity in the $x$ and $y$ coordinates.

The next section reviews some of the previous related work. In the following sections Fourier-based and smoothing-based techniques are applied to a particular meteorological data set. One finds that each of these techniques has its advantages, but that the standard errors of the velocity estimates appear notably smaller for the smoothing-based technique.

\subsection{Some History}

(Briggs, Phillips \& Shinn 1950) studied the behavior of radio waves reflected from the ionosphere. They were concerned with a randomly changing pattern moving across the ground. For example they were after drift velocities. Time series were envisaged recorded via an array of 3 receivers, i.e. at 3 locations $(x, y)$. With $Y(x, y, t)$ denoting the signal recorded at time $t$ and location $(x, y)$, the basic parameter suggested to be employed in the estimation was the correlation function

$$
\operatorname{corr}\{Y(x+v, y+w, t+u), Y(x, y, t)\}
$$

assumed not to depend on $x, y, t$. (Briggs 1968) extends the work and in particular assumes the function (2) has the form

$$
\rho\left[A\left(v-V_{x} u\right)^{2}+B\left(w-V_{y} u\right)^{2}+K u^{2}+2 H\left(v-V_{x} u\right)\left(w-V_{y} u\right)\right]
$$

with $\left(V_{x}, V_{y}\right)$ denoting the drift velocities. Estimates of the $V_{x}, V_{y}$ and the other parameters are obtained via nonlinear regression. (Briggs 1968) also discusses the case where dispersion occurs, that is the velocity depends on the wavenumber.

(Leese, Novak \& Clark 1971) study cloud motion via images taken from a geosynchronous satellite. They are interested in wind fields. Pictures, $Y(x, y, t)$, are taken at times $t=t_{1}$ and $t_{2}$. The basic criterion proposed is (2) with $t=t_{1}$ and $t+u=t_{2}$. The correlation is assumed to be independent of $(x, y)$ and the point, $(\hat{v}, \hat{w})$, of maximum cross-correlation is determined. The speed estimate is then $\sqrt{\hat{v}^{2}+\hat{w}^{2}} /\left(t_{2}-t_{1}\right)$ and the direction estimate $\tan ^{-1}(\hat{v} / \hat{w})$. These researchers found their procedure "Better

than manual for speed". They noted that complications that could lead to difficulties of estimation included: growth, decay, rotations and layers.

(Arking, Lo \& Rosenfeld 1978) take a Fourier approach. They suppose that 


$$
Y\left(x, y, t_{2}\right) \approx Y\left(x-v, y-w, t_{1}\right)
$$

The cross-spectrum of the two fields $Y\left(., t_{2}\right)$ and $Y\left(., t_{1}\right)$ is then given by

$$
f_{21}(\mu, \lambda) \approx e^{-i(\nu \mu+w \nu)} f_{11}(\mu, \nu)
$$

where $f_{11}($.$) denotes the spatial power spectrum of Y(x, y, t)$. The pair $(v, w)$ are estimated from neighboring wavenumber, $(\mu, \nu)$, data.

(Marshall 1980) was concerned with speed and direction of storm rainfall patterns. Data were available from a rain guage network, with sensors located at positions $\left(x_{j}, y_{j}\right), j=1,2, \ldots, J$ and measurements made at times $t=0,1,2, \ldots$. To carry through the analysis, the data were interpolated to a grid. This researcher also took a maximum cross-correlation approach, estimating for given $u$, the $(v, w)$ maximizing (2) above. If that extreme point is $\left(\hat{v}_{u}, \hat{w}_{u}\right)$, the mean velocity of the storm is estimated by the average of $\hat{v}_{u}$ and $\hat{w}_{u}$. (Marshall 1980) used least squares to fit the model

$$
h \exp \left\{-a^{2} r^{2}-b^{2} s^{2}\right\}
$$

to (2), with $r=v \sin \theta+w \cos \theta$ and $s=w \sin \theta-v \cos \theta$. (Brillinger 1985) indicated extensions of the results of (Hannan \& Thomson 1973) to provide large sample distributions for maximum cross-correlation estimates in the case of two time slices. (Brillinger 1993) is concerned with estimating the joint distributions of several successive motions given consecutive locations of moving particles. (Brillinger 1994) is concerned with the estimation of the travel times of the effects of cloud seeding. In that paper a conceptual model is built, analagous to that of (Le Cam 1961), for the transference of the effects, then both parametric and nonparametric estimation is carried out.

In the study of problems such as those just described, critical distinctions that arise include: Is the number of sensors, $J$, small or large? Is the velocity constant or dispersive? Is the number of time slices, $T$, small or large? The choices made affect the approximations to distributions of the estimates in important ways. When $J$ or $T$ are large, traditional asymptotic approximations are available.

References on the maximum cross-correlation approach are: (Burke 1987), (Kamachi 1989) and (Tokmakian, Strub \& McClean-Padman 1990). Estimation techniques, based on differential expressions of the motion, are reviewed in (Aggarwal \& Nandhakumar 1988). (Carroll, Hall \& Ruppert 1994) investigate penalized least squares and maximum cross-covariance methods for estimating the missalignment of a pair of images. Research continues on this type of problem, into the circumstances under which each method is to be preferred. 


\subsection{The Data}

The particular data studied here are a five day sequence of 0000 and 1200 Greenwich Mean Time (GMT) geopotential analyses. These are spatially interpolated estimates of the height of the 500 millibar (mb) pressure field across the surface of the Earth. This quantity provides the thickness of the atmosphere between the sea level and the $500 \mathrm{mb}$ level. It relates to temperature, being low for cold values and high for warm values. The data were prepared by the National Meteorological Center in Washington and one reference to the method is (Dey \& Morone 1985). The period covered is 1200 GMT 1 January 1986 to 0000 GMT 6 January 1986. The time interval between data is 12 hours and there are 10 time slices. The geopotential is computed on a 64 by 32 global grid, (64 equispaced longitudes and the 32 latitudes $85.8,80.3,74.7,69.2,63.7,58.1,52.6,47.1,41.5,36.0,30.5$, $24.5,19.4,13.8,8.3,2.8$ North and South). The data are based on many observations and are interpolated to this regular array. They are meant to provide input values for numerical forecasts in particular.

The measurements of 1200 GMT 1 January are graphed as contours in an image in Figure 1. Values 5300 meters and below are indicated by dashed lines. One sees, for example, a depression over Hudson Bay. Further examination of the 10 such images shows the depression to move eastward and fill in over the eastern Atlantic on 5 January.

\subsection{The Problem}

The problem of concern is how to estimate the velocity of a moving phenomenon, such as the $500 \mathrm{mb}$ field whose initial time slice is graphed in Figure 1. This field could be denoted $Y(x, y, t)$, but consideration will be restricted to motion along single latitudes. Denote the values along a given latitude, $y$, by

$$
Y(x, t)
$$

with $t$ refering to time and $x$ to longitude East. The model considered is

$$
Y(x, t)=g(x-v t)+\epsilon(x, t)
$$

with $\epsilon($.$) stationary noise. Here g($.$) represents the moving shape and v$ its velocity. Because the Earth is a sphere, the function $g($.$) has period 360^{\circ}$. Fields that are periodic are considered in (Yaglom 1962), (Monin 1963), (Hannan 1964), (DuFour \& Roy 1976). 


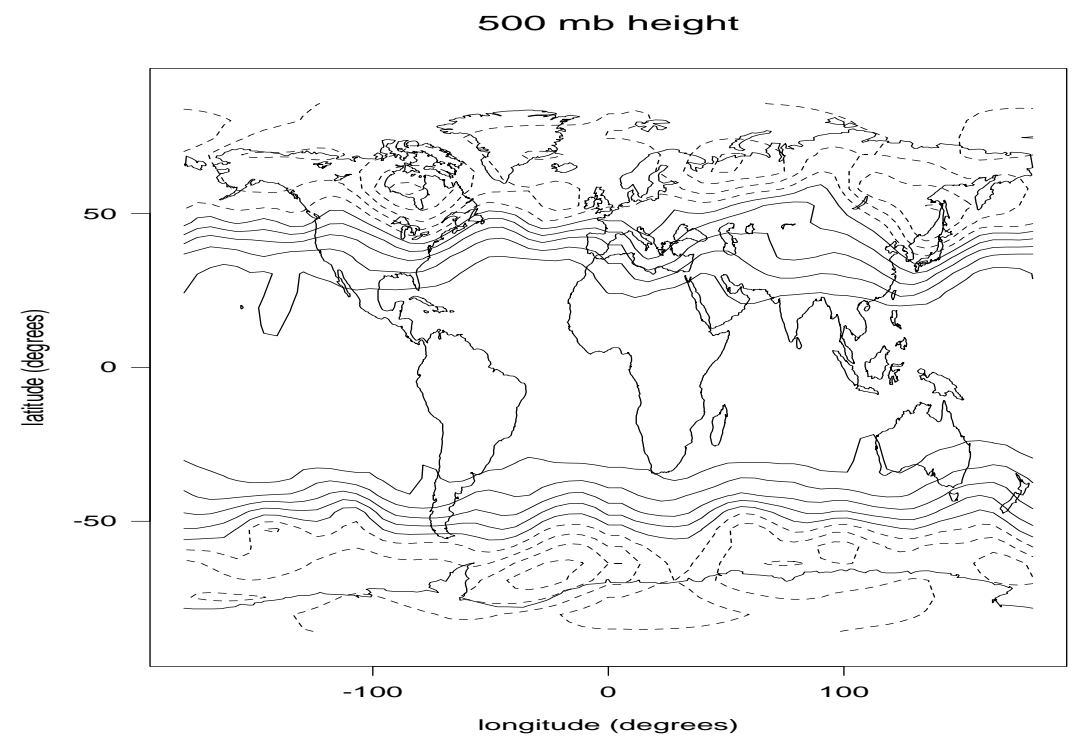

FIGURE 1.1. Contour plot of the $500 \mathrm{mb}$ height at 1200 GMT 1 January 1986. Contours at levels 5300 meters and below are indicated by dashed lines.Contours are spaced 100 meters apart.

\subsection{A Fourier Approach}

For the moment, let the longitude be expressed in radians, $\theta=2 \pi x / 360$, rather than degrees. To reduce the effects of the presence of a fixed or slowly moving disturbance, the process $Y($.) analyzed is that of the differences

$$
Y(x, t)=Z(x, t+1)-Z(x, t)
$$

with $Z($.$) the original 500 \mathrm{mb}$ values. The differencing operation enhances small features and makes the process values more nearly independent. Data are available for $\theta=2 \pi l / L, l=0, \ldots, L-1$ and $t=0, \ldots, T-1$. A first analysis will be based on the empirical Fourier transform

$$
d_{Y}^{T}(m, \lambda)=\sum_{l} \sum_{t} Y\left(\frac{2 \pi l}{L}, t\right) \exp \left\{-i \frac{2 \pi l m}{L}\right\} \exp \{-i \lambda t\}
$$

$L=64, T=9$. Suppose the Fourier series expansion of $g(\theta)$ is

$$
g(\theta)=\sum_{k} \beta_{k} \exp \{i \theta k\}
$$

$0 \leq \theta<2 \pi$ with $k$ wavenumber. Evaluating the Fourier transform of $g(\theta-v t)$ as in (4) one obtains 

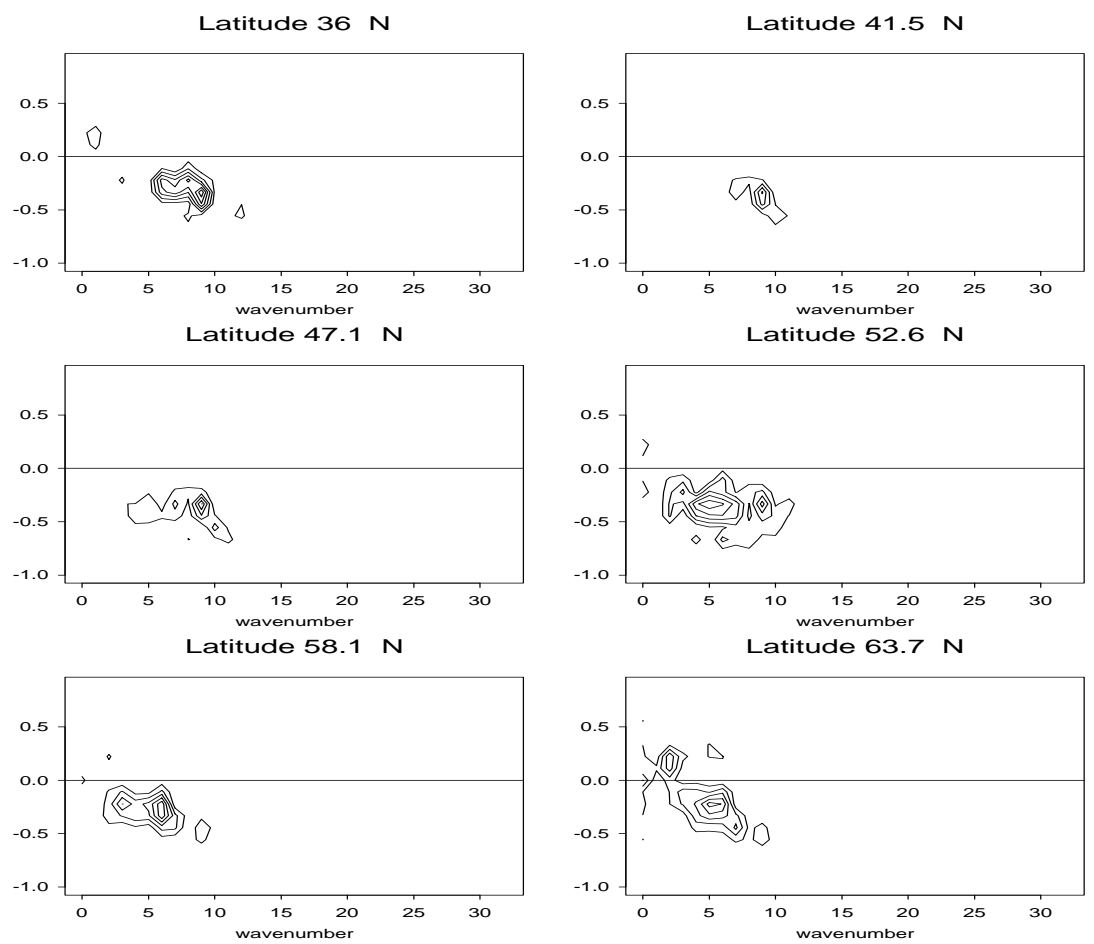

FIGURE 1.2. Lineal-temporal periodogram, $\left|d_{Y}^{T}(m, \lambda)\right|^{2}$, of the data values $Y(x, t)$ at 6 northern latitudes. Units of the $\mathrm{y}$-axis are cycles/day.

$$
\beta_{m} \Delta^{T}(\lambda+v m)
$$

with

$$
\Delta^{T}(\lambda)=\sum_{t=0}^{T-1} \exp \{-i \lambda t\}
$$

The function $\left|\Delta^{T}().\right|$ has principal mass near the origin, side lobes at $3 \pi / T, 5 \pi / T, \ldots$ and period $2 \pi$. By inspection $\left|d_{Y}^{T}(m, \lambda)\right|^{2}$, as a function of $m, \lambda$, can be anticipated to have a ridge along $\lambda+v m \approx 0$.

Figure 2 presents contour plots of $\left|d_{Y}^{T}(m, \lambda)\right|^{2}$, the lineal-temporal periodogram, for the 6 northern latitudes 36.0, 41.5, 47.1, 52.6, 58.1, 63.7 . Frequencies along the $y$-axis are in cycles/day. One sees principal peaks and suggestions of ridges, primarily at wavenumbers $5-10$ and periods of 3-10 days.

The quantity $d_{Y}^{T}(m, \lambda)$ is a double Fourier transform. In the estimation of velocity $v$, it is simpler to work with the following single Fourier transform 
Latitude $36 \mathrm{~N}$
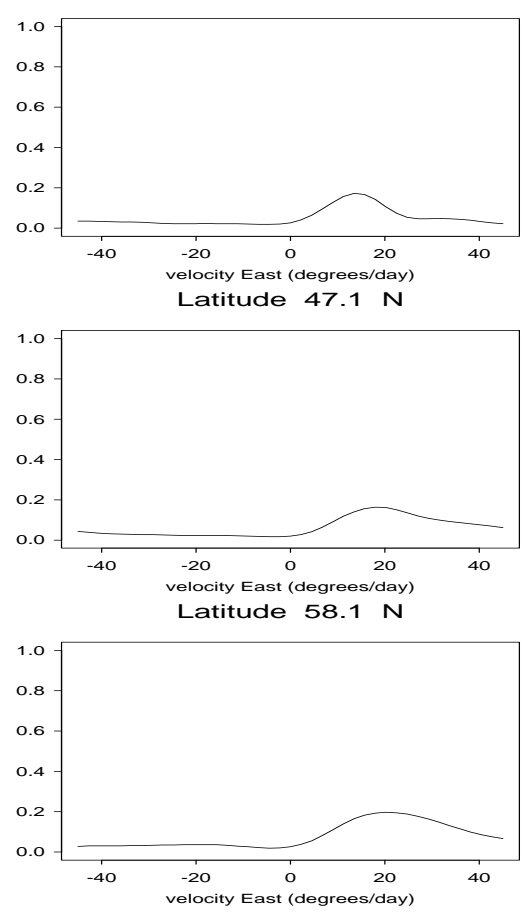

Latitude $41.5 \mathrm{~N}$
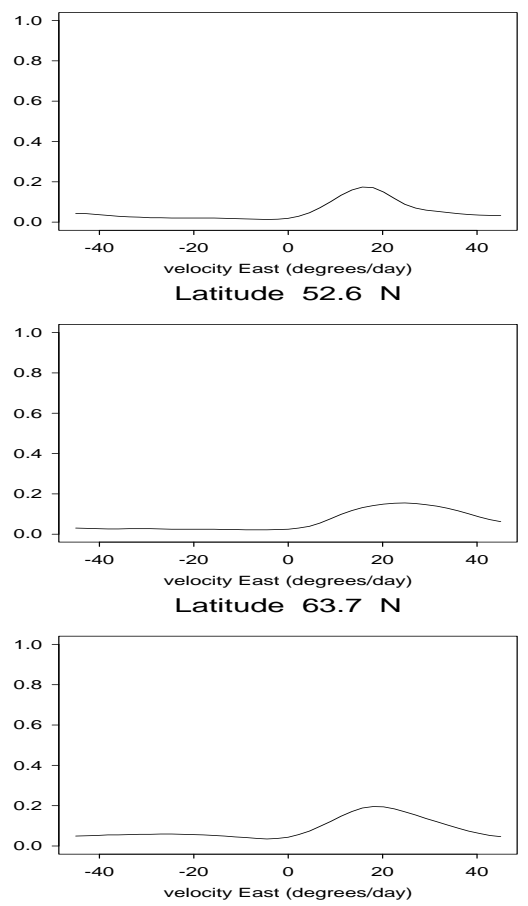

FIGURE 1.3. Plot of the multiple R-squared criterion (5) for the Fourier fitting procedure.

$$
Y_{m}(t)=\sum_{l} Y\left(\frac{2 \pi l}{L}, t\right) \exp \left\{-i \frac{2 \pi l m}{L}\right\}
$$

$t=0, \ldots, T-1$.

This is $\beta_{m} \exp \{-i v m t\}+$ noise under the model (3). Elementary regression suggests consideration of the multiple R-squared type statistic

$$
R(v)^{2}=1-\sum_{m}\left|\sum_{t} Y_{m}(t) e^{i v m t}\right|^{2} /\left(T \sum_{m} \sum_{t}\left|Y_{m}(t)\right|^{2}\right)
$$

as a measure of fit for a prespecified velocity value, $v$. To assess the optimal value of $v,(5)$ is graphed in Figure 3 taking $m=0, \ldots, 12$. (From Figure 2 the principal mass is at $m \leq 12$.) The maxima of (5) are seen to occur at the velocities near $20^{\circ}$ longitude/day. The largest value of the criterion is about .2 in each case and the peaks are fairly broad. This circumstance is reflected in the numerical estimates and associated uncertainties given in Section 1.7. 

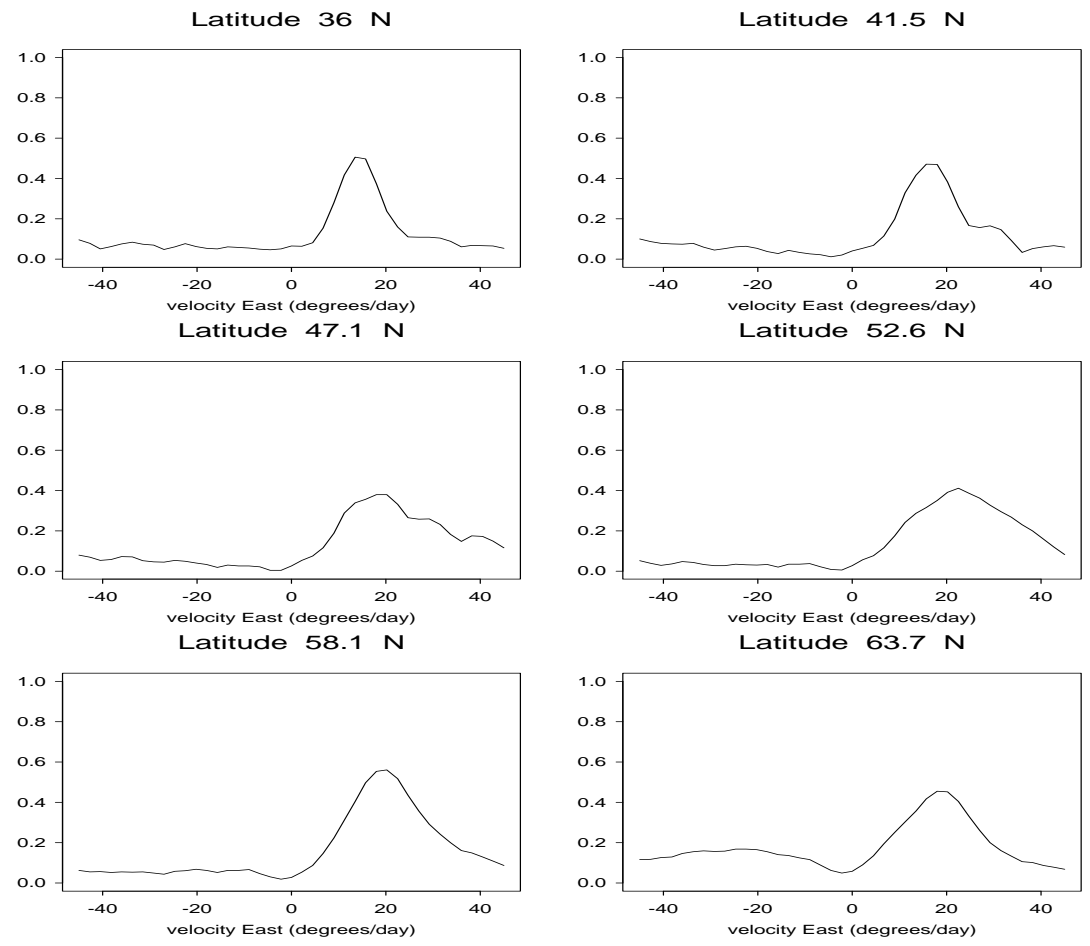

FIGURE 1.4. Plot of the multiple R-squared criterion (6) for the generalized additive model fitting procedure.

(Hayashi 1982) reviews space-time spectral analysis methods and their applications to large-scale atmospheric waves.

\subsection{A Nonparametric Approach}

Consider again the model (3). In the case that the velocity $v$ is known, but not $g(),.(3)$ is the simplest case of the generalized additive model, (Hastie \& Tibshirani 1990), (Hastie 1992). In the case of unknown $v$, it is the simplest case of projection pursuit regression, see (Friedman \& Stuetzle 1981). The function $g($.$) may therefore be estimated in a variety of manners,$ see the preceding references. In the present case, natural cubic splines with equi-spaced knots are employed, (Hastie \& Tibshirani 1990).

To start, $v$ is viewed as known. Then $g($.$) is estimated, based on the data$ values $(x-v t, Y(x, t))$. As a measure of fit, multiple R-squared

$$
R(v)^{2}=1-\sum(Y-\hat{g})^{2} / \sum(Y-\bar{Y})^{2}
$$



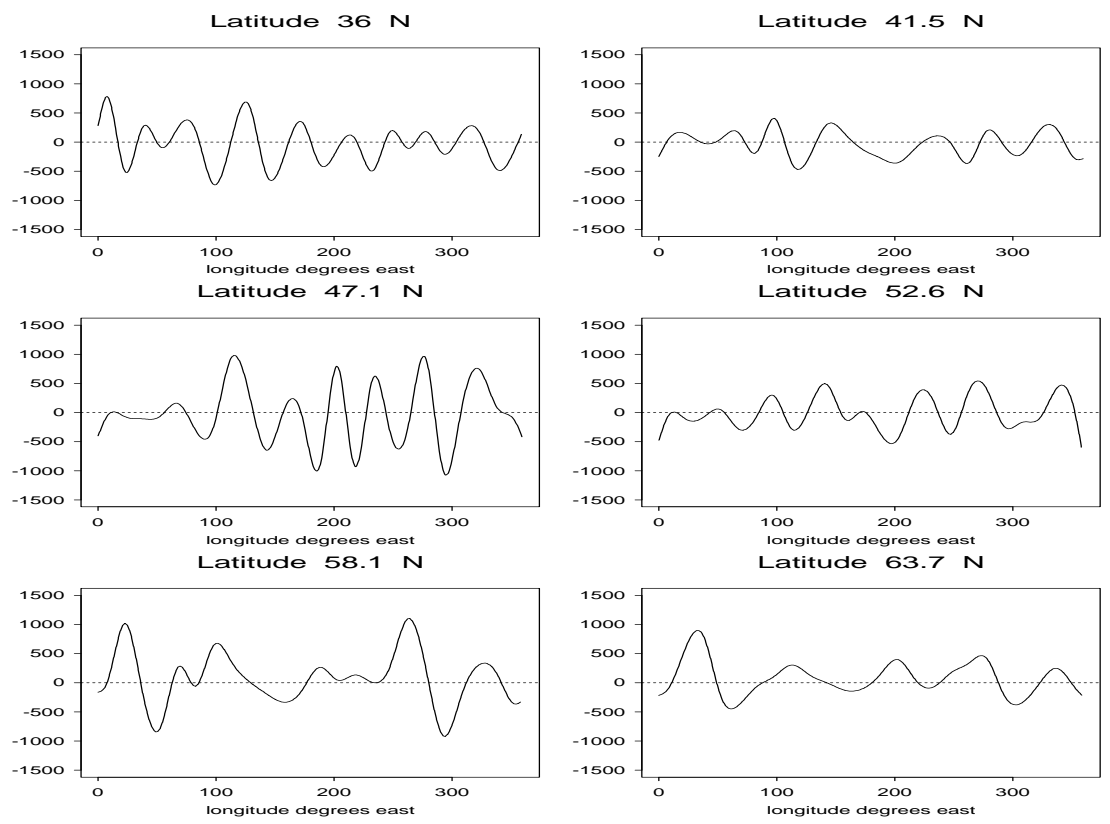

FIGURE 1.5. The estimated functions $\hat{g}(x)$ obtained via smoothing.

is again employed. Its values are graphed in Figure 4, as a function of $v$, for the 6 different latitudes. The peaks are much more prominent, but the maxima at the different latitudes are seen to occur at similar locations to those of Figure 3 .

Figure 5 graphs the estimates of the function $g($.$) for the 6$ latitudes.

In a search for periodicities, (Huber 1985) refers to such a technique as "A time series version of PPR (projection pursuit regression)".

\subsection{Uncertainty Estimation}

For the moment it will be assumed that the noise process, $\epsilon($.$) , consists$ of independent 0 mean common variance normals. To begin consider the Fourier procedure. The estimate maximizing (5) is maximum likelihood and there are classic formulas to approximate the standard error. In the present case, since the only parameter of concern is $v$, the simple procedure of (Richards 1961) may be employed. It has the advantage that one can disregard the nuisance parameters. It involves expressing the estimates of the $\beta$ 's, which are ordinary least squares, in terms of the parameter $v$ and after this substitution determining the maximizing value of $v$. Surprisingly the second derivative of this likelihood of the single variable, $v$, may be 
used to approximate the standard error of $\hat{v}$. The values obtained are:

\begin{tabular}{|c|c|c|}
\hline Latitude $^{\circ} N$ & velocity $^{\circ} /$ day & s.e. \\
\hline 36.0 & 14.6 & 28.4 \\
41.5 & 16.5 & 23.4 \\
47.1 & 17.4 & 31.2 \\
52.6 & 21.5 & 45.7 \\
58.1 & 19.2 & 33.8 \\
63.7 & 19.7 & 37.5 \\
\hline
\end{tabular}

These standard errors are large, compared to $\hat{v}$, as might have been expected from the broad peaks of Figure 3 .

In the case of fitting the model (3) with $g($.$) smooth, the results are:$

\begin{tabular}{|c|c|c|}
\hline Latitude $^{\circ} N$ & velocity $^{\circ} /$ day & s.e. $/$ day \\
\hline 36.0 & 13.9 & 5.9 \\
41.8 & 17.3 & 7.4 \\
47.1 & 18.7 & 14.0 \\
52.6 & 22.8 & 16.6 \\
58.1 & 19.4 & 10.4 \\
63.0 & 18.8 & 10.6 \\
\hline
\end{tabular}

These standard errors are notably smaller. This second procedure is apparently more sensitive and will perhaps pick up the fine features better. The spline employed had 24 nodes and hence 25 linear parameters. The Fourier technique also had 25 linear parameters.

\subsection{Discussion and Summary}

The results of the two analyses, the first a parametric Fourier and the second a nonparametric, are broadly similar. Advantages of the Fourier approach include: the model may also be examined by plots such as Figure 2 , periodicity is handled directly, the whiteness of the noise may be studied and autocorrelation may be introduced into the model as necessary. The advantages of the generalized additive model approach include: the estimates are (apparently) more precise and an estimate of $g($.$) is provided$ directly.

In interpreting the results one needs to keep in mind the possibility of aliasing, i.e. that there is a disturbance going around the planet so quickly that it appears to be moving slowly when sampled but every 12 hours.

The model can be extended to

$$
Z(x, t)=f(x)+g(x-\imath t)+\epsilon(x, t)
$$


and the "fixed" component $f($.$) estimated. As indicated in Section 1$ the model may also be extended to the case of several components moving with different velocities and directions, see (1). One could consider the automatic estimation of the smoothness and dimension parameters. Then the more general forms of generalized additive modelling and projection pursuit regression would be called for.

\subsection{Acknowledgements}

The $500 \mathrm{mb}$ geopotential data and helpful comments on it were provided by Francis Zwiers of Environment Canada. Alastair Scott pointed out the broad usefulness of (Richards 1961). The Editors gave important assistance. The initial nonparametric analyses were carried out via the ace procedure of (Breiman \& Friedman 1985). Preliminary versions of some of the material were presented in the Wilks Lectures, Princeton University May 1989, and at the 1990 Annual Meeting of the Statistical Society of Canada in St. John's, Newfoundland.

Enfin, je veux dire, "Merci beaucoup Professor Le Cam pour tout le conseil, statistique et personnel, pendant tant des années."

The research was supported in part by the National Science Foundation Grant MCS-9300002 and the Office of Naval Research Grant N00014-94-10042 .

\subsection{RefERENCES}

Aggarwal, J. K. \& Nandhakumar, N. (1988), 'On the computation of motion from sequences of images - a review', Proceedings of the IEEE pp. 917-935.

Arking, A., Lo, R. \& Rosenfeld, A. (1978), 'A Fourier approach to cloud motion estimation', Journal of Applied Meteorology pp. 735-744.

Breiman, L. \& Friedman, J. H. (1985), 'Estimating optimal transformations for multiple regression and correlation, (with discussion)', Journal of the American Statistical Association pp. $580-619$.

Briggs, B. (1968), 'On the analysis of moving patterns in geophysics - i. correlation analysis', Journal of Atmospheric and Terrestrial Physics 
pp. $1777-1794$.

Briggs, B., Phillips, G. \& Shinn, D. (1950), 'The analysis of observations on spaced receivers of the fading of radio signals', Proceedings of the Physical Society B pp. 106-121.

Brillinger, D. (1985), 'Fourier inference: some methods for the analysis of array and nonGaussian series data', Water Resources Bulletin pp. $743-756$.

Brillinger, D. R. (1993), 'Distributions of particle displacements via higher-order moment functions', IEE Proceedings-F pp. 390-394.

Brillinger, D. R. (1994), 'On a weather modification problem of Professor Neyman', Probability and Mathematical Statistics.

Burke, M. (1987), 'Moving random surfaces and correlation analysis', Radio Science pp. 607-624.

Carroll, R., Hall, P. \& Ruppert, D. (1994), 'Estimation of lag in misregistered, averaged images', Journal of the American Statistical Association pp. 219-229.

Cox, D. \& Isham, V. (1988), 'A simple spatial-temporal model of rainfall', Proceedings of the Royal Society A pp. 317-328.

Dey, C. \& Morone, L. (1985), 'Evolution of the national meteorological center global data assimilation system: January 1982 - December 1983', Monthly Weather Review pp. 304-318.

DuFour, J.-M. \& Roy, R. (1976), 'On spectral estimation for a homogeneous random process on the circle', Stochastic Processes and their Applications pp. 107-120.

Friedman, J. \& Stuetzle, W. (1981), 'Projection pursuit regression', Journal of the American Statistical Association pp. 817-823.

Gupta, V. \& Waymire, E. (1987), 'On Taylor's hypothesis and dissipation in rainfall', Journal of Geophysics Research pp. 9657-9660.

Hannan, E. (1964), 'The statistical analysis of hydrological time series', Proceedings of the National Symposium on Water Resources pp. $233-243$.

Hannan, E. \& Thomson, P. (1973), 'Estimating group delay', Biometrika pp. 241-252.

Hastie, T. (1992), Generalized additive models In Statistical Models in $S$ (eds. J.M. Chambers and T.J. Hastie), Wadsworth, Pacific Grove, Wadsworth. Pacific Grove. 
Hastie, T. \& Tibshirani, R. (1990), Generalized Additive Models, Chapman and Hall. London.

Hayashi, Y. (1982), 'Space-time analysis and its applications to atmospheric waves', Journal of the Meteorological Society of Japan pp. $156-171$.

Huber, P. J. (1985), 'Projection pursuit', Annals of Statistics pp. 435-475.

Kamachi, M. (1989), 'Advective surface velocities derived from sequential images for rotational flow field: limitations and applications of maximum cross correlation method with rotational registration', Journal of Geophysical Research pp. 18227-18233.

Le Cam, L. M. (1961), A stochastic description of precipitation Proceedings of the Fourth Berkeley Symposium on Mathematical Statistics and Probability (ed. J. Neyman), University of California Press. Berkeley.

Leese, J., Novak, C. \& Clark, B. (1971), 'An automated technique for obtaining cloud motion from geosynchronous satellite data using cross correlation', Journal of Applied Meteorology pp. 118-132.

Marshall, R. (1980), 'The estimation and distribution of storm movement and storm structure, using a correlation analysis technique and rain-guage data', Journal of Hydrology pp. 19-39.

Monin, A. (1963), Stationary and periodic time series in the general circulation of the atmosphere Time Series Analysis (ed. $M$. Rosenblatt), Wiley. New York.

Phelan, M. (1992), Aging functions and their nonparametric estimation in point process models of rainfall In Statistics in the Environmental and Earth Sciences (eds. A.T. Walden and P. Guttorp), Arnold. London.

Richards, F. (1961), 'A method of maximum-likelihood estimation', Journal of the Royal Statistical Society B pp. 469-475.

Smith, J. \& Karr, A. (1985), 'Parameter estimation for a model of space-time rainfall', Water Resources Research pp. 1251-1257.

Tokmakian, R., Strub, P. \& McClean-Padman, J. (1990), 'Evaluation of the maximum cross-correlation method of estimating sea surface velocities from sequential satellite images', Journal of Atmospheric and Oceanic Technology pp. 852-865.

Yaglom, A. (1962), An Introduction to the Theory of Stationary Random Functions, Prentice Hall. New York. 